\title{
Global and Local Contrast Adaptive Enhancement for Non-uniform Illumination Color Images
}

\author{
Qi-Chong Tian and Laurent D. Cohen \\ Université Paris-Dauphine, PSL Research University, \\ CNRS, UMR 7534, CEREMADE, \\ 75016, Paris, France \\ \{tian, cohen\}@ceremade.dauphine.fr
}

\begin{abstract}
Color images captured by digital devices may contain some non-uniform illuminations. Many enhancement methods produce undesirable results in the aspect of contrast improvement or naturalness preservation. A global and local contrast enhancement method is proposed for adaptively enhancing the non-uniform illumination images. Firstly, a novel global contrast adaptive enhancement algorithm obtains the global enhancement image. Secondly, a huepreserving local contrast adaptive enhancement algorithm produces the local enhancement image. Finally, a contrastbrightness-based fusion algorithm obtains the final result, which represents a trade-off between global contrast and local contrast. This method improves the visual quality and preserves the image naturalness. Experiments are conducted on a dataset including different kinds of non-uniform illumination images. Results demonstrate the proposed method outperforms the compared enhancement algorithms both qualitatively and quantitatively.
\end{abstract}

\section{Introduction}

Contrast enhancement is an important low-level computer vision technique, which can improve the visibility of the considered images. This kind of algorithm has been widely applied in camera photographing, video quality improvement, medical imaging, and remote sensing imaging [17]. Moreover, it is usually used for improving the image quality in some other computer vision applications, such as object segmentation [30], object recognition [12], and high dynamic range image rendering [28].

Due to the non-uniform lighting conditions, imaging device limitations or unsuitable exposure parameter settings, the acquired images need to be improved the contrast and the brightness. There are some existing contrast enhancement methods [8] to deal with this problem. However, these algorithms usually obtain undesirable enhancement results, including over enhancement in the bright regions, under enhancement in the dark regions, or producing unnaturalness artifacts.

In this paper, we propose a global and local contrast adaptive enhancement method, which can improve the contrast and preserve the naturalness of the considered image. The proposed method is composed of three stages. Firstly, a global contrast adaptive enhancement method is conducted on the original non-uniform illumination image. Then, a local contrast adaptive enhancement method is applied to obtain the corresponding locally enhanced image. At last, the final enhanced image is obtained via a contrast-brightnessbased fusion algorithm. The main contributions of the proposed algorithm include presenting a novel global adaptive contrast enhancement method, considering hue preservation in the enhancement framework, and developing a contrastbrightness-based fusion method.

The rest of this paper is organized as follows. The related work is briefly summarized in Section (2). The detailed description of the proposed method is presented in Section (3). The numerical experiments and performance evaluations are shown in Section (4). Finally, we give the conclusions and the future work in Section (5).

\section{Related Work}

There are a large number of image enhancement algorithms, in which histogram-based methods and filter-based methods are received the most attentions.

Histogram-based methods. One of the most popular histogram-based enhancement methods is histogram equalization [8], which modifies the original histogram to a uniform histogram for obtaining higher contrasts. This method may produce undesirable artifacts, such as overenhancement. Various related methods [13, 32] have been proposed to avoid these disadvantages. Arici et al. [3] presented a general histogram equalization framework for con- 
trast enhancement via optimizing cost functions. Rivera et al. [29] applied adaptive mapping functions to obtain well-enhanced images, which spread the dynamic range of the dark intensities and maintain the shapes of their histograms. Lee et al. [16] proposed an enhancement algorithm based on the layered difference reproduction of histograms. Contrast-limited adaptive histogram equalization [26] is another popular histogram-based enhancement method, in which the histogram equalization is considered locally. This kind of local methods [20,5] can improve more details in the considered image, and preserve the image naturalness. Stark [31] proposed an adaptive image contrast enhancement method based on a generalization of histogram equalization. By setting different forms of mapping functions, this algorithm can obtain corresponding different degrees of contrast enhancement.

Filter-based methods. Homomorphic filter [23] is a very useful nonlinear tool for improving the image contrast and preserving the image detail information. Unsharp masking-based enhancement algorithms [27, 6] improve the contrasts for the high-frequency component and the lowfrequency component respectively. Jmal et al. [10] combined the mapping curve and the modified homomorphic filter to produce higher contrast images. This method obtains good results having a trade-off between the contrast improvement and naturalness preservation. Inspired by the retinex theory [15], there are many retinex-based contrast enhancement algorithms, which essentially are filter-based methods. Considering a Gaussian kernel to model the relation between the center pixel and surround pixels, Jobson et al. [11] proposed a classic retinex-based enhancement. This method has been well improved by Petro et al. [24] recently. Kimmel et al. [14] proposed a variational model for the retinex enhancement via a quadratic programming optimization. Morel et al. [19] explored some new alternative kernels for different kinds of enhancement applications. Pierre et al. [25] proposed a hue-preserving perceptual contrast enhancement model with a variational framework, which can void over-enhancement.

\section{Global and Local Contrast Adaptive En- hancement}

The overall framework of the proposed global and local contrast adaptive enhancement method is illustrated in Fig. (1). The considered non-uniform illumination color image $X_{c}$ is enhanced by a global method and a local method respectively. Then the globally enhanced color image $G_{c}$ and the locally enhanced color image $L_{c}$ are fused to produce a well-enhanced color image $Y_{c}$. The detailed description of the proposed method is presented below.

\subsection{Global Contrast Adaptive Enhancement}

\subsubsection{Overview}

We present the overview of the novel global contrast adaptive enhancement method, which is shown in Fig. (2). The hue preservation is also analyzed in this part.

Many color image enhancement methods treat the three color channels separately, which will change the hue of the original images. This kind of methods often generate some unnatural looking images, so hue preservation is an important technique for the color enhancement algorithms. There are different definitions of hue. Since HSI (HueSaturation-Intensity) [8] color space is a common color model in many computer vision applications, the definition of hue in HSI model is adopted in our method. Giving a single RGB color pixel $p$ with values $(r, g, b)$, the hue of $p$ is defined by Eq. (1).

$$
h u e(p)= \begin{cases}0 & \text { if } r=g=b, \\ \theta & \text { if } b \leq g, \\ 2 \pi-\theta & \text { if } b>g,\end{cases}
$$

where

$$
\theta=\arccos \frac{\frac{1}{2}((r-g)+(r-b))}{\left((r-g)^{2}+(r-b)(g-b)\right)^{\frac{1}{2}}} .
$$

Proposition 1. The single pixel $p_{1}$ with values $\left(r_{1}, g_{1}, b_{1}\right)$ $\in[0, L-1]^{3}$ and the other single pixel $p_{2}$ with valuse $\left(r_{2}, g_{2}, b_{2}\right) \in[0, L-1]^{3}$ have the same hue if and only if there exist $a, d \in \mathbb{R}$ such that $\left(r_{1}, g_{1}, b_{1}\right)=a\left(r_{2}, g_{2}, b_{2}\right)+d \mathbf{1}_{3}{ }^{T}$, where $\mathbf{1}_{3}:=(1,1,1)^{T}$, $L$ is the range of pixel values, $L=256$ for 8-bit images. The proof of this proposition can be found in [7].

The above proposition is a basic theory on hue preservation. Hue-preserving-based enhancement methods are analyzed in [21, 22], which demonstrate this kind of method is better than the traditional channel-by-channel enhancements or methods only enhancing the intensity channel. In our global contrast adaptive enhancement method, the pixel value range of a color image $X_{c}$ is firstly converted to the full range $[0, L-1]$ with the linear stretching [2] via Eq. (2).

$$
\hat{X}_{c}=(L-1) \frac{X_{c}-X_{\min }}{X_{\max }-X_{\min }},
$$

where $X_{\min }$ and $X_{\max }$ are respectively the minimal and the maximal intensity values among the three color channels of the image $X_{c}$, and $\mathrm{L}=256$ for 8-bit images.

The stretched color image $\hat{X}_{c}$ is converted to the corresponding intensity image $I$ using Eq. (3).

$I=0.299 \cdot \hat{X}_{R}+0.587 \cdot \hat{X}_{G}+0.114 \cdot \hat{X}_{B}$, 


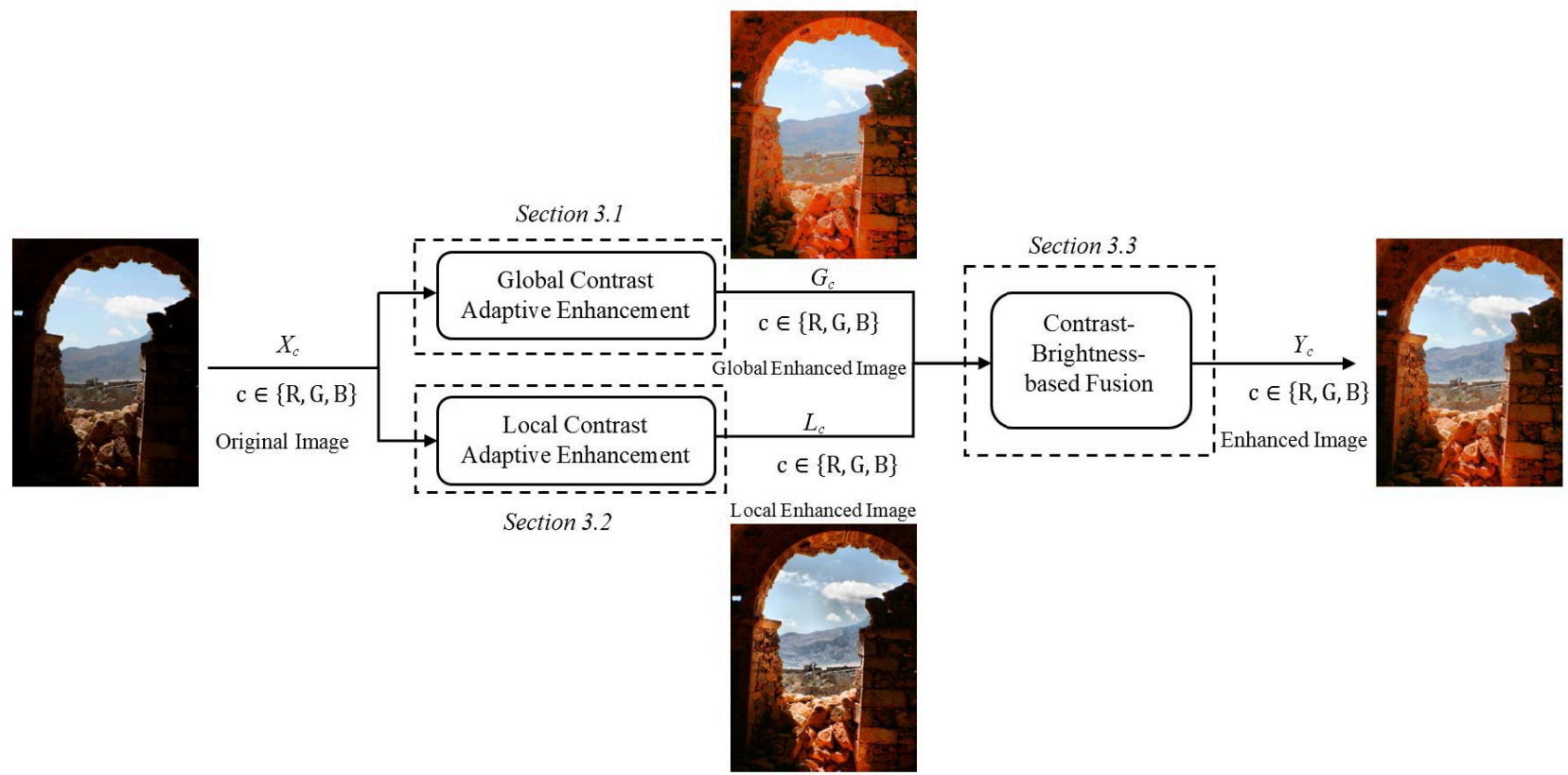

Figure 1. The overall framework of the proposed enhancement method. The original image $X_{c}$ has low contrast. The global enhanced image $G_{c}$ has good global contrast, however the detailed structure information is not desirable in some regions. The local enhanced image $L_{c}$ increased the detailed structure information, however the visual quality is not desirable. The final enhanced image $Y_{c}$ represents a trade-off between the global contrast and the local contrast.

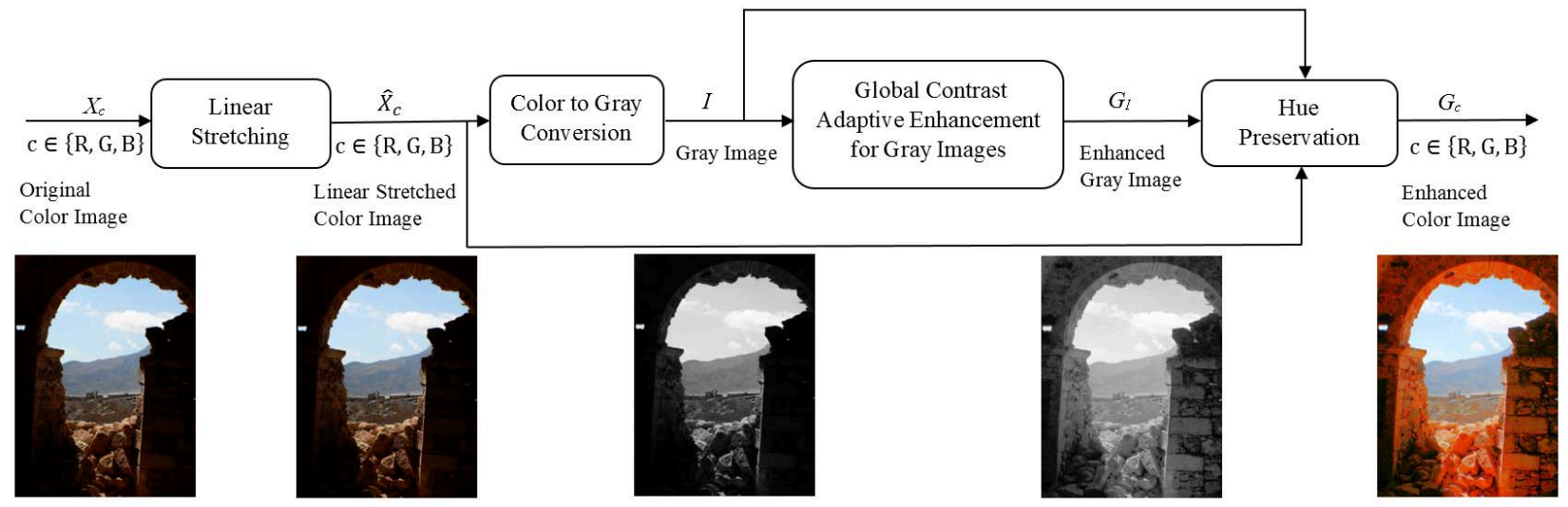

Figure 2. The overview of the global contrast adaptive enhancement method.

where $\hat{X}_{R}, \hat{X}_{G}, \hat{X}_{B}$ are the three color channels of the stretched color image $\hat{X}$.

Then a novel contrast enhancement algorithm is applied to $I$ to obtain the corresponding enhanced intensity image $G_{I}$. At last, we use the hue preservation enhancement framework to obtain the final enhanced color image $G_{c}$ with Eq. (4).

$G_{c}(k)=\left\{\begin{array}{lr}\frac{G_{I}(k)}{I(k)} \hat{X}_{c}(k), & \text { if } \frac{G_{I}(k)}{I(k)}<=1, \\ \frac{L-1-G_{I}(k)}{L-1-I(k)}\left(\hat{X}_{c}(k)-I(k)\right)+G_{I}(k), & \text { if } \frac{G_{I}(k)}{I(k)}>1,\end{array}\right.$

where $c \in\{R, G, B\}$ represents the corresponding color channel, and $k$ is the indexes of pixels in each color channel.
Nikolova et al. [22] have analyzed and proved the efficiency of the hue preservation with this equation.

\subsubsection{Global Contrast Adaptive Enhancement for Gray Images}

In this section, we consider a gray image $I$, with a total number of $N$ pixels and an intensity level range of $[0, L-1]$. The normalized histogram $h_{I}$ of the image $I$ is computed by Eq. (5).

$$
h_{I}(s)=\frac{n_{s}}{N}
$$


where $s \in[0, L-1]$ is pixel values, and $n_{s}$ is the total number of pixels having the same value $s$.

Histogram-based contrast enhancement methods use the image histogram to obtain the corresponding mapping function for modifying the pixel values and enhancing the contrast of the considered image. Traditional histogram equalization algorithm uses the original histogram of the considered image to obtain the mapping function. The mapping function $T$ for the image $I$ is given by Eq. (6)

$$
T(i)=\left\lfloor(L-1) \sum_{j=0}^{i} h_{I}(j)+0.5\right\rfloor,
$$

where $i \in[0, L-1]$ is the input integer for the mapping function, and $\rfloor$ computes the nearest integer of the considered value towards minus infinity.

Traditional histogram equalization methods try to create a uniform histogram for the enhanced image via considering a cumulative histogram as the corresponding mapping function. This kind of histogram equalization algorithm often produces unnatural looking images with visual artifacts. To deal with this problem, a general histogram modification framework is proposed in [3]. Using the mapping function obtained from the optimal modified histogram, the contrastenhanced image can avoid unnatural artifacts.

The modified histogram $h$ should be closer to the normalized uniform histogram $h_{U}$ and the value of the residual $h-h_{I}$ should be also small. The problem of obtaining the optimal modified histogram $\tilde{h}$ can be regarded as a solution of a bi-criteria optimization problem, which can be formulated as a weighted sum of two terms as Eq. (7).

$$
\min \left(\left\|h-h_{I}\right\|+\lambda\left\|h-h_{U}\right\|\right),
$$

where $\left\|h-h_{I}\right\|$ is a norm of $h-h_{I},\left\|h-h_{U}\right\|$ is a norm of $h-h_{U}$, and the parameter $\lambda>0$ adjusts the trade-off between the contrast enhancement and the data fidelity. An analytical solution of Eq. (7) can be obtained when the squared sum of the $L_{2}$ norm is adopted. This problem can be rewritten in Eq. (8).

$$
\tilde{h}=\underset{h}{\operatorname{argmin}}\left(\left\|h-h_{I}\right\|_{2}^{2}+\lambda\left\|h-h_{U}\right\|_{2}^{2}\right) .
$$

This is a quadradic optimization problem, and the solution of Eq. (8) is given by Eq. (9).

$$
\tilde{h}=\left(\frac{1}{1+\lambda}\right) h_{I}+\left(\frac{\lambda}{1+\lambda}\right) h_{U} .
$$

The optimal modified histogram $\tilde{h}$ is a weighted sum of $h_{I}$ and $h_{U}$. This is a simple and efficient solution to obtain the modified histogram. The parameter $\lambda$ needs to be carefully tuned for obtaining satisfied enhancement results. Different values of $\lambda$ can produce different modified histograms, thus generate different contrast enhanced images.

Our goal is to adaptively enhance the contrast of the considered image. To deal with this problem, we adopt the tone distortion of the mapping function $T$ to guide the optimization. The tone distortion measure [33] is defined by Eq. (10).

$D(T)=\max _{0 \leq j \leq i \leq L-1}\left\{i-j ; T(i)=T(j), h_{I}(i)>0, h_{I}(j)>0\right\}$.

In this definition, we can know that the smaller the tone distortion $D(T)$ the smoother the tone reproduced by the mapping function $T$. The smoother tone means less unnatural looking in the enhanced image. In other words, the small tone distortion can avoid unnatural artifacts produced by the contrast enhancement algorithm. The tone distortion measure $D$ is obtained from the mapping function $T$, which is computed with the optimally modified histogram $\tilde{h}$. And the histogram $\tilde{h}$ depends on the weighted parameter $\lambda$. So we can use the tone distortion to select the optimal weighted parameter $\lambda$, which can produce the optimal modified histogram and the final mapping function. This mapping function can be utilized to adaptively enhance images.

\subsection{Local Contrast Adaptive Enhancement}

The proposed global contrast adaptive enhancement method can improve the global contrast and brightness of the whole image. However, it is essentially a global method, which may reduce the local contrast or the detail information in the original image. So we consider a local contrast adaptive enhancement to improve the local contrast and preserve the detail information.

In this method, we combine a hue preservation enhancement framework in the Eq. (4) and the Contrast-Limited Adaptive Histogram Equalization method (CLAHE) [26] to improve the local contrast and preserve the hue of the considered image. The overall framework of this method is similar to the framework shown in Fig. (2). The only difference is that contrast-Limited Adaptive Histogram Equalization takes the place of Global Contrast Adaptive for Gray Images. The detailed description of CLAHE can be found in the original paper [26].

\subsection{Contrast-Brightness-based Fusion}

Inspired by the idea of exposure fusion [18] and fusionbased dehazing [1], we develop a contrast-brightness-based fusion framework to obtain the final enhanced image $Y_{c}$ with the globally enhanced image $G_{c}$ and the locally enhanced image $L_{c}$. This fusion framework can keep the better contrast and brightness for each pixel, since we adopt contrast-brightness-based pixel-level weights to fuse every pixel. Firstly, the weight maps of the globally enhanced image and the locally enhanced image are computed by Eq. 
(11).

$$
W_{d}=\min \left\{C_{d}, B_{d}\right\}, \quad d \in\{G, L\},
$$

where $W_{d}$ is the weight map, $C_{d}$ is the contrast measure, $B_{d}$ is the brightness measure, and the operation min can efficiently penalize the corresponding low-contrast, low brightness (under-exposure) or high brightness (over-exposure). $C_{d}$ is obtained by a Laplacian filter, which can assign high weights to edges and textures in the corresponding image. $B_{d}$ is computed by a Gaussian curve $\exp \left(-\frac{(i-0.5)^{2}}{2 \sigma^{2}}\right)$ and $\sigma=0.2$ in our experiments, which can assign high weights to pixel values close to 0.5 and define low weights to pixel values near 0 (under-exposure) or near 1 (over-exposure). Secondly, the two weight maps are normalized by Eq.(12) to ensure a consistent fusion result.

$$
\hat{W}_{d}=\frac{W_{d}}{W_{G}+W_{L}}, \quad d \in\{G, L\},
$$

Finally, the fusion result is obtained by Eq. (13).

$Y_{c}(k)=\hat{W}_{G}(k) \cdot G_{c}(k)+\hat{W}_{L}(k) \cdot L_{c}(k), c \in\{R, G, B\}$,

where $k$ is the indexes of pixels in each color channel. Due to the hue-preserving framework analyzed in Section (3.1.1), we only compute the weight maps for the intensity images of the globally enhanced color image and locally enhanced color image, and then apply them to the three color channels. The results computed directly via Eq. (13) usually contain the undesired seam problem in the fused image, so we adopt a Laplacian pyramid method [4] to avoid this problem. Burt et al. [4] utilized this technique to seamlessly blend two images at multiple resolutions using a pyramidal image decomposition. Multiple resolutions blending is effective for avoiding seams, because it blends features instead of intensities.

\section{Experiments and Comparisons}

We ran the proposed method and several state-of-the-art image enhancement methods on a dataset including 25 nonuniform illumination color images. These test images are widely used in the literature. The comparison algorithms include Rivera et al.'s content-aware enhancement via channel division (Rivera'12) [29] , Lee et al.'s layered difference representation contrast enhancement (Lee'13) [16] , Petro et al.'s improved multiscale retinex contrast enhancement (Petro'14) [24] , Jmal et al.'s enhancement method by saturation feedback and homomorphic filter (Jmal'17) [10], Arici et al.'s histogram-modification-based global contrast enhancement (Arici'09) [3], and the Contrast-Limited Adaptive Histogram Equalization method (CLAHE) [26]. The source codes of Rivera'12, Lee'13, Jmal'17 are available on the authors' homepages. The source code and

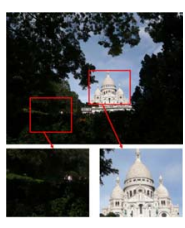

(a) Original

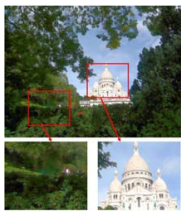

(e) Jmal'17

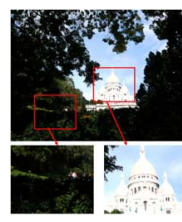

(b) Rivera' 12

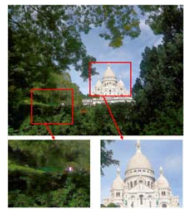

(f) Arici’09

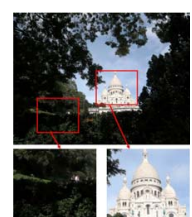

(c) Lee'13

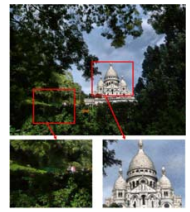

(g) CLAHE

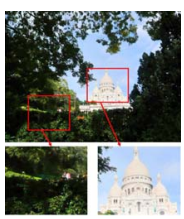

(d) Petro'14

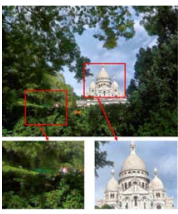

(h) Proposed
Figure 3. Results of the image Sacré-Cœur. (a). Original image; (b)-(h). Results enhanced respectively by Rivera'12 [29], Lee'13 [16], Petro'14 [24], Jmal'17 [10], Arici'09 [3], CLAHE [26] and the proposed method. For the visual comparison, the areas in the red rectangles are enlarged and shown below the corresponding images.

online demonstration of Petro' 14 are available on the official website of the IPOL Journal. CLAHE is implemented by the Matlab Image Processing Tool function adapthisteq. We reproduced the method of Arici'09 (For all experiments, the parameter $\lambda=1$ ).

To evaluate the performance of the proposed adaptive enhancement method, both qualitative comparisons and quantitative assessments are considered in the following parts.

\subsection{Qualitative Comparisons}

In this part, we show some original test images and the corresponding enhanced images to subjectively compare the performances of these enhancement algorithms. The contrast enhancement and naturalness preservation are considered in the comparisons.

Fig. (3) shows the enhanced results on the image Sacré-Cœur (Fig. 3(a)), which contains the high illumination on the building and the low illumination on the tree. Our goal is to enhance the dark regions (low illuminations) and the bright regions (high illuminations), without reducing the details in the bright regions. Rivera'12 (Fig. 3(b)) slightly enhances the dark region and over-enhances the bright region, which obtains the worst result. Lee'13 (Fig. 3(c)) slightly enhances the dark region and preserves the bright region. Petro'14 (Fig. 3(d)) obtains the good result in the dark region, however over-enhances the bright region. Arici'09 (Fig. 3(f)) enhances the global contrast very well, however reduces the local contrast in the bright region. CLAHE (Fig. 3(g)) enhances the local contrast well, however the whole visual quality is unsatisfied. Jmal'17 (Fig. 3(e)) and the proposed method (Fig. 3(h)) produce good results both in the dark region and the bright region. From the 
enlarged bright regions, we can easily observe that the proposed method obtains better contrasts in the bright regions than the results of Jmal'17. Our method generates good results both in the dark regions and in the bright regions, resulting from combining the globally enhanced image and the locally enhanced image. Moreover, the hue preservation framework can avoid the over-enhancement problem to some extent.

Some other results are shown in Fig. (4), Fig. (5) and Fig. (6), which demonstrate the proposed method can obtain good results both in the dark regions and the bright regions. The other algorithms either slightly enhance the dark regions or over-enhance the bright areas, which produce undesirable enhanced results.

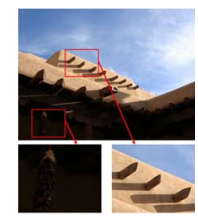

(a) Original

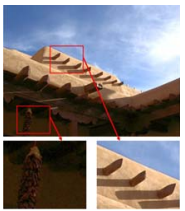

(e) Jmal'17

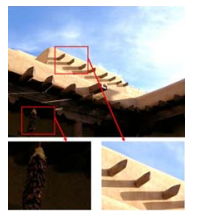

(b) Rivera' 12

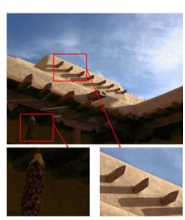

(f) Arici'09

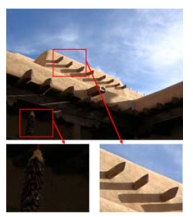

(c) Lee' 13

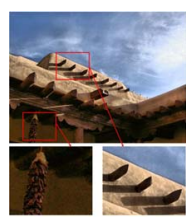

(g) CLAHE

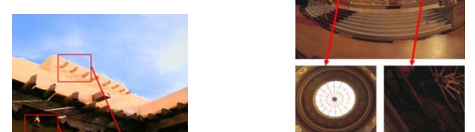

(e) Jmal'17

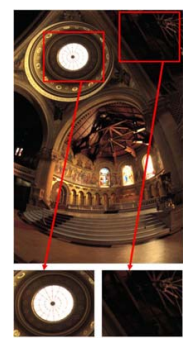

(b) Rivera'12

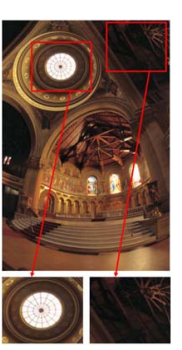

(f) Arici'09

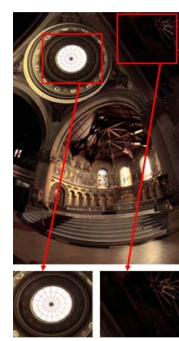

(c) Lee'13

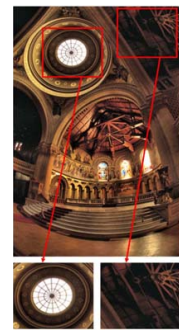

(g) CLAHE

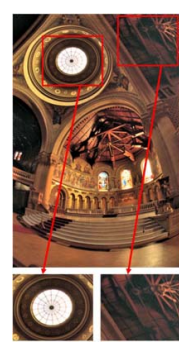

(d) Petro' 14

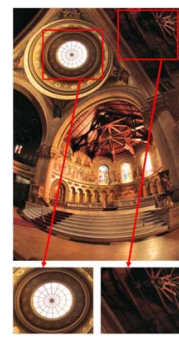

(h) Proposed
Figure 5. Results of the image Church. (a). Original image; (b)-(h). Results enhanced respectively by Rivera'12 [29], Lee'13 [16], Petro'14 [24], Jmal'17 [10], Arici'09 [3], CLAHE [26] and the proposed method. Rivera' 12 and Lee' 13 both failed in the dark region and the bright region, Petro' 14 obtained the good contrast in the dark region and slightly enhanced the bright region, Jmal' 17 reduced the sharpness in the bright region, Arici'09 obtained the good contrast in the bright region and slightly enhanced the dark region, CLAHE did not obtain good result in the bright region, the proposed method produced the acceptable contrast in the bright region and in the dark region.

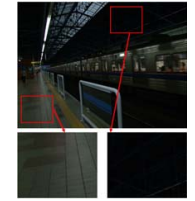

(a) Original

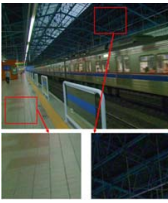

(e) Jmal'17

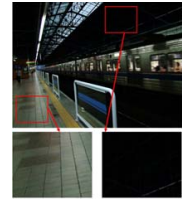

(b) Rivera' 12

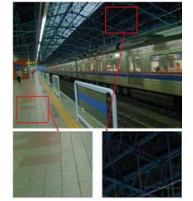

(f) Arici'09

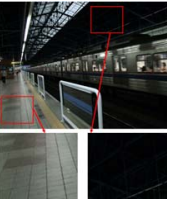

(c) Lee'13

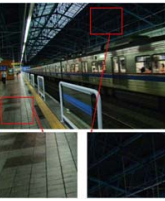

(g) CLAHE

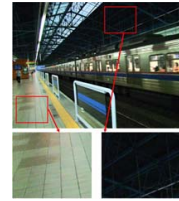

(d) Petro' 14

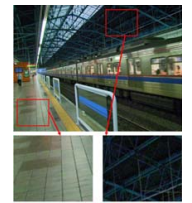

(h) Proposed a contrast evaluation method via Visible Edges Assessment (VEA). This metric evaluates the contrast improvement with three indicators. The first indicator $e$ evaluates the ability for restoring edges. The second indicator $\bar{r}$ is the mean ratio of gradients in visible edges. The third indicator $\Sigma$ represents the percentage of pixels becoming saturated after the enhancement. The higher values of $e$ and $\bar{r}$ present the better contrast improvement. The lower value of $\Sigma$ indicates the better ability of pixel value range preservation. The evaluation results are shown in Table (1), which demon-

Figure 6. Results of the image Metro. (a). Original image; (b)-(h). Results enhanced respectively by Rivera'12 [29], Lee'13 [16], Petro'14 [24], Jmal'17 [10], Arici'09 [3], CLAHE [26] and the proposed method. Rivera'12 and Lee'13 failed in the dark region, Petro' 14 slightly enhanced the dark region, Jmal'17 and Arici'09 reduced the sharpness in the bright region, CLAHE did not produce good visual quality, the proposed method produced good contrasts in the dark region and the bright region. 
strates CLAHE and the proposed method outperforms other algorithms in terms of contrast improvement (higher $e$ and $\bar{r}$ ) and pixel value range preservation (lower $\Sigma$ ). The local processing of CLAHE and the fusion processing of the proposed method can generate more visible edges. Since more visible edges may result in unnatural looking images, a comparison of the image naturalness is also considered in this quantitative analysis.

Yeganeh et al. [34] proposed a Statistical Naturalness Measure (SNM) model for evaluating the image quality. They consider the global intensity distributions of a large number of images and build a model to assess the image naturalness. The range of SNM is $[0,1]$. The higher value of SNM means the better naturalness of the considered image. We use this metric to evaluate our method and several compared algorithms on the test dataset. The results are shown in Table (2), which indicates that the proposed method outperforms other algorithms in terms of the image naturalness. The fusion model and the hue preservation framework can ensure our method obtain better results with higher naturalness.

\subsection{Computation Cost}

The proposed method contains the global adaptive contrast enhancement with optimization processing, the hue preservation contrast-limited adaptive histogram equalization, and the contrast-brightness-based image fusion framework. We implemented the function of the proposed method in Matlab without improving the code performance. The dataset includes 25 color images. Each color image contains around 200 thousands pixels. The total running time is 20.25 seconds and the average processing time for one image is 0.81 seconds. The code performance will be improved with $\mathrm{C}$ programming and parallel computing.

\section{Conclusions}

In this paper, we have proposed a global and local contrast adaptive enhancement method for non-uniform illumination color images. There are three main contributions of our method. Firstly, a novel global adaptive contrast enhancement method is proposed to improve the global contrast. Secondly, a hue preservation framework is considered in our algorithm. Thirdly, a contrast-brightness-based image fusion method is developed for obtaining final enhanced images. Experiments demonstrate the proposed method outperforms other enhancement methods in terms of subject visual comparisons and objective evaluations.

Since there are undesirable noises in the dark regions of some non-uniform illumination images, noise reductions will be considered in the contrast enhancement framework in the future work.

\section{References}

[1] C. O. Ancuti and C. Ancuti. Single image dehazing by multi-scale fusion. IEEE Transactions on Image Processing, 22(8):3271-3282, 2013. 5

[2] C. O. Ancuti, C. Ancuti, and P. Bekaert. Enhancing by saliency-guided decolorization. In Computer Vision and Pattern Recognition (CVPR), 2011 IEEE Conference on, pages 257-264. IEEE, 2011. 2

[3] T. Arici, S. Dikbas, and Y. Altunbasak. A histogram modification framework and its application for image contrast enhancement. IEEE Transactions on Image Processing, 18(9):1921-1935, 2009. 2, 4, 5, 6

[4] P. Burt and E. Adelson. The Laplacian pyramid as a compact image code. IEEE Transactions on Communications, 31(4):532-540, 1983. 5

[5] V. Caselles, J.-L. Lisani, J.-M. Morel, and G. Sapiro. Shape preserving local histogram modification. IEEE Transactions on Image Processing, 8(2):220-230, 1999. 2

[6] G. Deng. A generalized unsharp masking algorithm. IEEE Transactions on Image Processing, 20(5):1249-1261, 2011. 2

[7] J. H. Fitschen, M. Nikolova, F. Pierre, and G. Steidl. A variational model for color assignment. In International Conference on Scale Space and Variational Methods in Computer Vision (SSVM'15), volume LNCS9087, pages 437448. Springer, May 2015. 2

[8] R. C. Gonzalez and R. E. Woods. Digital image processing (third edition). Englewood Cliffs, NJ, USA: Prentice-Hall, 2007. 1, 2

[9] N. Hautière, J.-P. Tarel, D. Aubert, and E. Dumont. Blind contrast enhancement assessment by gradient ratioing at visible edges. Image Analysis \& Stereology, 27(2):87-95, 2011. 6

[10] M. Jmal, W. Souidene, and R. Attia. Efficient cultural heritage image restoration with nonuniform illumination enhancement. Journal of Electronic Imaging, 26(1):011020011020, 2017. 2, 5, 6

[11] D. J. Jobson, Z.-u. Rahman, and G. A. Woodell. A multiscale retinex for bridging the gap between color images and the human observation of scenes. IEEE Transactions on Image processing, 6(7):965-976, 1997. 2

[12] W.-C. Kao, M.-C. Hsu, and Y.-Y. Yang. Local contrast enhancement and adaptive feature extraction for illuminationinvariant face recognition. Pattern Recognition, 43(5):17361747, 2010. 1

[13] Y.-T. Kim. Contrast enhancement using brightness preserving bi-histogram equalization. IEEE Transactions on Consumer Electronics, 43(1):1-8, 1997. 2

[14] R. Kimmel, M. Elad, D. Shaked, R. Keshet, and I. Sobel. A variational framework for retinex. International Journal of computer vision, 52(1):7-23, 2003. 2

[15] E. H. Land and J. J. McCann. Lightness and retinex theory. Journal of the Optical Society of America, 61(1):1-11, 1971. 2

[16] C. Lee, C. Lee, and C.-S. Kim. Contrast enhancement based on layered difference representation of 2D histograms. 


\begin{tabular}{cccccccc}
\hline & Rivera'12 & Lee'13 & Petro'14 & Jmal'17 & Arici'09 & CLAHE & Proposed \\
\hline$e$ & 0.0033 & 0.0759 & -0.0331 & 0.0125 & 0.0200 & $\mathbf{0 . 2 0 3 3}$ & $\mathbf{0 . 0 8 7 2}$ \\
$\bar{r}$ & 1.6179 & 1.6376 & $\mathbf{2 . 7 7 4 5}$ & 2.2258 & 2.2686 & 2.7030 & $\mathbf{2 . 9 3 7 0}$ \\
$\Sigma(\%)$ & 2.4371 & 1.6536 & 1.0296 & 1.4663 & 0.0441 & $\mathbf{0 . 0 0 1 9}$ & $\mathbf{0 . 0 2 7 0}$ \\
\hline
\end{tabular}

Table 1. Average VEA of enhancement algorithms on the test dataset

\begin{tabular}{ccccccccc}
\hline & Original & Rivera'12 & Lee'13 & Petro'14 & Jmal'17 & Arici'09 & CLAHE & Proposed \\
\hline SNM & 0.1977 & 0.3732 & 0.3429 & $\mathbf{0 . 5 0 5 2}$ & 0.4883 & 0.4727 & 0.4487 & $\mathbf{0 . 6 6 6 8}$ \\
\hline \multicolumn{7}{c}{ Table 2. Average SNM of enhancement algorithms on the test dataset }
\end{tabular}

IEEE Transactions on Image Processing, 22(12):5372-5384, 2013. 2, 5, 6

[17] J.-L. Lisani, J. Michel, J.-M. Morel, A. B. Petro, and C. Sbert. An inquiry on contrast enhancement methods for satellite images. IEEE Transactions on Geoscience and Remote Sensing, 54(12):7044-7054, 2016. 1

[18] T. Mertens, J. Kautz, and F. Van Reeth. Exposure fusion: A simple and practical alternative to high dynamic range photography. In Computer Graphics Forum, volume 28, pages 161-171, 2009. 5

[19] J.-M. Morel, A. B. Petro, and C. Sbert. What is the right center/surround for retinex? In IEEE International Conference on Image Processing (ICIP'14), pages 4552-4556. IEEE, 2014. 2

[20] W. M. Morrow, R. B. Paranjape, R. M. Rangayyan, and J. L. Desautels. Region-based contrast enhancement of mammograms. IEEE Transactions on Medical Imaging, 11(3):392406, 1992. 2

[21] S. K. Naik and C. Murthy. Hue-preserving color image enhancement without gamut problem. IEEE Transactions on Image Processing, 12(12):1591-1598, 2003. 2

[22] M. Nikolova and G. Steidl. Fast hue and range preserving histogram specification: Theory and new algorithms for color image enhancement. IEEE Transactions on Image Processing, 23(9):4087-4100, 2014. 2, 3

[23] A. V. Oppenheim, R. Schafer, and T. Stockham. Nonlinear filtering of multiplied and convolved signals. IEEE Transactions on Audio and Electroacoustics, 16(3):437-466, 1968. 2

[24] A. B. Petro, C. Sbert, and J.-M. Morel. Multiscale retinex. Image Processing On Line, pages 71-88, 2014. 2, 5, 6

[25] F. Pierre, J.-F. Aujol, A. Bugeau, G. Steidl, and V.-T. Ta. Variational contrast enhancement of gray-scale and RGB images. Journal of Mathematical Imaging and Vision, 57(1):99-116, 2017. 2

[26] S. M. Pizer, E. P. Amburn, J. D. Austin, R. Cromartie, A. Geselowitz, T. Greer, B. ter Haar Romeny, J. B. Zimmerman, and K. Zuiderveld. Adaptive histogram equalization and its variations. Computer Vision, Graphics, and Image Processing, 39(3):355-368, 1987. 2, 4, 5, 6

[27] A. Polesel, G. Ramponi, and V. J. Mathews. Image enhancement via adaptive unsharp masking. IEEE Transactions on Image Processing, 9(3):505-510, 2000. 2

[28] E. Reinhard, W. Heidrich, P. Debevec, S. Pattanaik, G. Ward, and K. Myszkowski. High dynamic range imaging: acquisi- tion, display, and image-based lighting. Morgan Kaufmann, 2010. 1

[29] A. R. Rivera, B. Ryu, and O. Chae. Content-aware dark image enhancement through channel division. IEEE Transactions on Image Processing, 21(9):3967-3980, 2012. 2, 5, 6

[30] G. Schaefer, M. I. Rajab, M. E. Celebi, and H. Iyatomi. Colour and contrast enhancement for improved skin lesion segmentation. Computerized Medical Imaging and Graphics, 35(2):99-104, 2011. 1

[31] J. A. Stark. Adaptive image contrast enhancement using generalizations of histogram equalization. IEEE Transactions on Image Processing, 9(5):889-896, 2000. 2

[32] Y. Wang, Q. Chen, and B. Zhang. Image enhancement based on equal area dualistic sub-image histogram equalization method. IEEE Transactions on Consumer Electronics, 45(1):68-75, 1999. 2

[33] X. Wu. A linear programming approach for optimal contrasttone mapping. IEEE Transactions on Image Processing, 20(5):1262-1272, 2011. 4

[34] H. Yeganeh and Z. Wang. Objective quality assessment of tone-mapped images. IEEE Transactions on Image Processing, 22(2):657-667, 2013. 7 